infection at baseline. Efficacy data were synthesised using a DerSimonian and Laird weighted random-effect model. The mean odds ratio (OR) and 95\% confidence interval (CI) for the association between Cervarix $^{\mathrm{TM}}$, Gardasil ${ }^{\oplus}$ and HPV-16 monovalent vaccine and HPV-associated cervical intraepithelial neoplasia grade 3 or worse (CIN3+) was 0.90 (CI: $0.56,1 \cdot 44)$ and for the association between Gardasil $^{\circledR}$ and HPV-associated vulval/vaginal intraepithelial neoplasia grades 2-3 (VIN2-3/VaIN2-3) OR 1.20 (CI: 0·07, 20·40).

Conclusion There was no evidence that the HPV vaccines are effective in preventing vaccine-type HPV-associated pre-cancer in women with evidence of prior HPV exposure in this analysis. However, these studies were not designed to investigate the efficacy in this group, so statistical power (sample size, follow-up period and event rate) was insufficient to detect a small effect size. Longer follow-up is also needed to detect possible prevention of re-infection.

\section{P3.373 MALE CIRCUMCISION PREVALENCE, KNOWLEDGE, PERCEPTIONS, AND INTENT AMONG MEN IN BULAWAYO, ZIMBABWE: A CROSS-SECTIONAL STUDY}

doi:10.1136/sextrans-2013-051184.0826

$1,2 \mathrm{Z}$ A Kaufman, ${ }^{3} \mathrm{~J}$ DeCelles, ${ }^{3} \mathrm{~K}$ Bhauti, ${ }^{1} \mathrm{H}$ A Weiss, ${ }^{4} \mathrm{C} N$ Chaibva, 'D A Ross. 'London School of Hygiene and Tropical Medicine, London, UK; ${ }^{2}$ Wits Reproductive Health and HIV Institute, Johannesburg, South Africa; ${ }^{3}$ Grassroot Soccer, Bulawayo, Zimbabwe, ${ }^{4}$ National University of Science and Technology, Bulawayo, Zimbabwe

Background Zimbabwe has a target to reach $80 \%$ voluntary medical male circumcision (VMMC) coverage among HIV-negative 15-29 year-old men by 2015. This is a central strategy in the nation's HIV response. Despite considerable recent investment, uptake has been slower than hoped. A cluster-randomised trial began in 2012 to assess the effectiveness of a sport-based VMMC demand-creation intervention.

Methods At baseline, 663 men aged 18-45 years (median age 24 years) on 47 local soccer teams (both social and professional) in Bulawayo completed a self-administered questionnaire on VMMCrelated knowledge, perceptions and intent using touchscreen mobile phones. Linear and logistic regressions were used to assess differences by age, educational attainment, and study group, adjusting for team-level clustering.

Results 141 men $(21.0 \%)$ reported being circumcised, the majority $(80.6 \%)$ at a hospital or clinic and $24(17.0 \%)$ within the last three months. Among the uncircumcised men, the majority (90.8\%) knew that VMMC reduces HIV risk and thought that getting circumcised was a good idea (89.3\%). About half (54.2\%) correctly identified at least one local clinic providing VMMC services and $62.6 \%$ reported that they were planning to get circumcised. Among uncircumcised men, those with A-level/higher education had better VMMC knowledge ( $\mathrm{AOR}=3.15,95 \% \mathrm{CI}=1.52-6.53)$, but were less likely to intend to become circumcised ( $\mathrm{AOR}=0.57,95 \% \mathrm{CI}=0.37-0.89$ ). Being circumcised was weakly associated with having A-Level/higher education (AOR $=1.52,95 \% \mathrm{CI}=0.95-2.43$ ). No differences were observed between study groups in reported circumcision status, age, education, VMMC knowledge, or VMMC intention.

Conclusion This study provides evidence that VMMC-related knowledge and intentions are high amongst uncircumcised, soccerplaying men in Bulawayo, though VMMC coverage remains far below $80 \%$. Effective demand creation interventions are needed and should ensure uncircumcised men are aware of local sites offering VMMC services. Further research should investigate barriers to VMMC uptake among men in Bulawayo.

\section{P3.374 TREATMENT FAILURE HAS IMPORTANT IMPLICATIONS FOR CHLAMYDIA TRANSMISSION AND THE EFFECTIVENESS OF SCREENING PROGRAMIMES}

doi:10.1136/sextrans-2013-051184.0827
'D G Regan, 'D P Wilson, '2J S Hocking. 'Kirby Institute, University of New South UK, Sydney, Australia; 'Centre for Women's Health, Gender and Society, University of Melbourne, Melbourne, Australia

Background It is generally considered that current treatment regimens for chlamydia treatment are highly effective, achieving a cure rate of around $97 \%$. Some recent studies, however, suggest that treatment failure may occur at a rate that is substantially higher than previously thought.

Methods We use a mathematical transmission model to estimate the population-level impact of treatment failure on chlamydia transmission and on the effectiveness of screening strategies in reducing chlamydia prevalence. We assume treatment failure rates ranging from $3 \%$ (baseline) to a maximum of $23 \%$ in the context of female-only and female-plus-male screening programmes where between $15 \%$ and $50 \%$ annual screening coverage is achieved. We examine the impact that increased treatment failure may have on prevalence and on the time and screening coverage required to achieve specific reductions in prevalence.

Results Based on sexual mixing patterns and health-seeking behaviour for young Australians, the model predicts that population prevalence would almost double, from $\sim 4.5 \%$ to $\sim 8 \%$, if treatment failure increased from $3 \%$ to $23 \%$. To compensate for higher assumed treatment failure, relative increases in screening coverage of between $\sim 4 \%$ and $\sim 16 \%$ will be required to achieve a reduction of $50 \%$ in chlamydia prevalence within 5 years under the treatment failure scenarios evaluated. The time required for screening to deliver equivalent reductions in prevalence as predicted under the baseline treatment failure rate is predicted to increase by between $\sim 6 \%$ and $\sim 35 \%$ (relatively) if the assumed treatment failure rate is increased to between $8 \%$ and $23 \%$, depending on the screening strategy (female-only or female-plus-male) and the duration of screening (5 or 10 years).

Conclusion The rate of treatment failure may have a significant impact on the screening coverage and time required to achieve target reductions in chlamydia prevalence. This should be carefully considered when evaluating the potential effectiveness of proposed screening programmes.

\section{P3.375 EFFECTIVE USE OF MOBILE PHONES IN HIV PREVENTION IN UGANDA}

doi:10.1136/sextrans-2013-051184.0828

C 0 Wanyana, S Nambafu, E Musoke Seruma, D Mpiima. The AIDS Support Organization(TASO) Uganda Limited, Jinja, Uganda

Background Uganda currently has a population of about 7 million people who own mobile phones.

There is always a challenge of reaching out to big populations of people with HIV prevention services.

Mobile phone usage is sited as one of the effective tools in reducing costs and improving efficiency in reaching out to people who need HIV prevention services.

Program Description The Aids Support Organization (TASO) Uganda Limited, uses mobile phones to follow up their clients on HIV treatment, management and for planning psychosocial support visits to client's family.

Partnerships with Media Telecom companies to send bulk or group SMS massages like reminders of wedding meetings sent to very many different people at the same time and less costs.

Lessons learnt It's cost effective to use mobile phones to reach a big numbers of people in the community for HIV prevention Conclusion Effective partnership with Telecom companies will improve on reaching out to mobile users in HIV prevention. TASO to continue using mobile phones to improve on their community programmes. 